\title{
Assessment of targeted selective treatment criteria to control subclinical gastrointestinal nematode infections on sheep farms
}

\author{
Carlos Calvete $^{\mathrm{a}, *}$, José M. González ${ }^{\mathrm{b}}$, Luis M. Ferrer ${ }^{\mathrm{c}}$, Juan J. Ramos ${ }^{\mathrm{c}}$, Delia Lacasta ${ }^{\mathrm{c}}$, \\ Ignacio Delgado ${ }^{a}$, Joaquín Uriarte ${ }^{\mathrm{a}}$ \\ a Animal Production and Health Department, Agrifood Research and Technology Centre of Aragon (CITA), Agrifood Institute of Aragon-IA2 (CITA-Zaragoza University), \\ Avda. Montañana, 930, 50059, Zaragoza, Spain \\ ${ }^{\mathrm{b}}$ Animal Pathology Department, Veterinary Faculty, University of Zaragoza, 50013, Zaragoza, Spain \\ ${ }^{\mathrm{c}}$ Animal Pathology Department, Veterinary Faculty, University of Zaragoza, Agrifood Institute of Aragon-IA2 (CITA-Zaragoza University), 50013, Zaragoza, Spain
}

\section{A R T I C L E I N F O}

\section{Keywords:}

Productive efficiency

Small ruminants

Subclinical gastrointestinal nematodosis

Targeted selective treatments

\begin{abstract}
A B S T R A C T
Control of sheep gastrointestinal nematode (GIN) infections mostly relies on the use of anthelmintics. Refugiabased control strategies as targeted selective treatments (TST) can delay anthelmintic resistance development, but the optimal decision criteria for selecting individuals to be treated in subclinical infection scenarios remain unclear. The present study aimed to evaluate the suitability of body condition score (BCS) and faecal egg counts (FEC) as treatment indicators and to determine their optimized threshold values for treatment in TST by determining the relationships of BCS, FEC and anthelmintic treatment with several productive parameters in premating and pre-partum periods at an individual level. Deworming in pre-mating period increased BCS gain, but its magnitude was directly associated with strongyle FEC before treatment. Deworming also increased fertility in ewes with BCS $<2.75$ and the proportion of lambing ewes that got pregnant during the first ovulation cycle. Nevertheless, treatment did not yield productive benefits in ewes with higher BCS values. On the other hand, deworming in the pre-partum lowered lamb peri-partum mortality and increased lamb weight at birth and the growth of lambs during lactation, especially in lambs born from ewes with strongyle FEC > 400 epg before treatment. These results showed that FEC and especially BCS can be potential decision criteria for the implementation of TST in these types of scenarios. The TST scheme derived from the present results on the control of GIN infections should include anthelmintic treatment of ewes with BCS lower than $\approx 3$ approximately five weeks before mating and lambing. In the pre-lambing period, treatment may be unnecessary if flock mean strongyle FEC is lower than $\approx 200 \mathrm{epg}$, as the proportion of ewes with individual FEC $>400$ epg would be very low. The results suggest that implementation of this TST scheme would provide benefits, such as the improvement of productivity, a rational management of parasites in refugia, and preservation of future efficacy of anthelmintics, in comparison to traditional deworming schemes.
\end{abstract}

\section{Introduction}

Gastrointestinal nematode (GIN) infections are a major cause of economic losses in ruminant livestock production, primarily through subclinical disease, highlighting the need to improve parasite management in sheep (Charlier et al., 2014a; Mavrot et al., 2015). Control of GINs relies mostly on the use of anthelmintics (Kenyon and Jackson, 2012; Valvárcel et al., 2013); however, anthelmintic resistance (AR) may develop in GIN populations from intensive applications (Rose et al., 2015). Since the rate of development of AR could be slowed by maintaining a proportion of the parasite population in refugia (unexposed to anthelmintic) (Van Wyk, 2001), treatment approaches targeting both the flock and the individual have been introduced to promote the sustainable use of anthelmintics. In targeted treatments (TT), the whole group is treated on the basis of knowledge of the risk or parameters that quantify the severity of infection, whereas in targeted selective treatments (TST), only individual animals within the group are treated to provide epidemiological and/or production benefits (Kenyon and Jackson, 2012).

Both treatment approaches, especially TST, are based on determining realistic thresholds for single or combined pathophysiological and/or production-based treatment indicators, such as clinical signs, body condition score (BCS), faecal egg count (FEC), weight gain or milk yield (Bath and van Wyk, 2009; Charlier et al., 2014a).

\footnotetext{
* Corresponding author.

E-mail address: ccalvete@aragon.es (C. Calvete).
} 
Nevertheless, the suitability of treatment indicators and the results obtained on health and productivity of animals have varied depending on the parasitological scenario, the production system and the technical resources available (Charlier et al., 2014a) and, in addition, because thresholds for treatment have been holistically or empirically established in many surveys, with no previous study or calibration of relationships among treatment indicators and parasite-sheep productivity (e.g., Kenyon et al., 2013; Torres-Acosta et al., 2014; Valcárcel et al., 2015). This situation indicates the need for suitable indicators, which should be identified, and whose threshold values for treatment should be calibrated to area, productive system and even to farm-specific conditions to increase their efficacy (Chylinski et al., 2015).

In south-western Europe, GINs mainly produce subclinical infections in sheep farms, and most farmers continue applying anthelmintic strategies based on systematic treatments in spring and/or autumn without any supporting diagnostic, epidemiological knowledge or awareness of the effect of deworming on sheep productivity (Calvete et al., 2012; Valvárcel et al., 2013, 2015). In this area, AR, although present, has not yet reached the worrying levels reported in other areas of the world; therefore, farmers are reluctant to assimilate changes from this traditional deworming scheme (Calvete et al., 2012; MartínezValladares et al., 2013).

In this context, the goal of the present work was twofold. In the first instance, the work aimed to determine the suitability of BCS and FEC as treatment indicators for TST in low-medium challenge environments, since both indicators can be easily estimated. In the second, the present work evaluated, at an individual level, the relationships between both indicators and the interactions among parasites, deworming and reproductive sheep efficiency in order to set threshold values for treatment that optimize the trade-off between sheep productivity and TST implementation.

\section{Materials and methods}

\subsection{Animal management}

The study was conducted on the sheep flock belonging to the Agrifood Research and Technology Centre of Aragon (CITA), located in Zaragoza Province, Northeast Spain. The flock comprised 590 ewes individually identified with intraruminal electronic boluses. Three different genotypic groups were present: 437 ewes belonged to the Rasa Aragonesa breed (R), 70 belonged to the prolific variant of Rasa Aragonesa $\left(\mathrm{ROA}^{\circledR}\right)$ (Martínez-Royo et al., 2008), and 83 were Rasa Aragonesa $x$ Romanov (F1). The age of the ewes ranged from 0.8 to 10.9 years old during the survey.

During the study, the flock grazed simultaneously in irrigated and non-irrigated fenced private pastures, with no other flocks grazing in the same areas. The flock was raised for meat production under a semiintensive management system representative of the ovine breeding activity in the region; however, productive schedules (mainly timing of mating) were usually modified and adapted to cover the needs of experimental assays performed with lambs in CITA.

Breeding was mainly performed by natural mating with Rasa Aragonesa rams. In a few cases, however, depending on the requirements of the experimental assays, artificial insemination with semen from the same breed was used. On all occasions, sheep heat was synchronised by using vaginal sponges impregnated with flugestone acetate (Syncro-part ${ }^{\circledR}$ sponge, CEVA lab., Barcelona, Spain) and the administration of PMSG hormone (Syncro-part ${ }^{\circledR}$ PMSG, CEVA lab., Barcelona, Spain). Approximately five weeks before insemination or the start of the mating period, the nutritional regime of the ewes was modified by administration of a concentrated feed mixture to bring out a better body condition at mating. For natural mating, ewes were placed with rams for approximately 35 days, and after that, rams were separated from the ewes, with pregnant ewes remaining together in the same batch until weaning.
Ewes were maintained outdoors in pastures throughout the year, but from one month before the start of lambing, ewes were again supplemented with a concentrate feed mixture, and 15 days before the start of lambing, they were housed in facilities with concrete soil and maintained only with hay and concentrate until weaning, when the lambs, between 40-60 days old, were moved to commercial fattening units or used in experimental assays in CITA.

\subsection{Experimental design}

All animal experiments were performed in compliance with the provisions of Spanish national and European laws (Law 32/2007, modified 6/2013, and RD 53/2013) and approved by the CITA Ethical Committee for Animal Experimentation (protocol 2017-06).

From April 2015 to July 2017, 1113 mating cases (209 by artificial insemination) were surveyed from mating to parturition and lamb weaning. Mating cases were distributed in ten mating batches conducted in spring ( 5 batches), autumn ( 3 batches) and winter ( 2 batches). The number of ewes per batch ranged from 67 to 187 animals, and the three genotypic groups were present in all batches.

Three sampling periods were set: the pre-mating period, pre-partum period and from parturition to weaning of lambs.

\subsubsection{Sampling procedures}

Pre-mating sampling was conducted five weeks before the rams were introduced into the mating batch. Faecal samples, collected directly from the rectum, were obtained from all the ewes and kept refrigerated before being processed the next day. Faecal samples were examined individually by using a modification of the McMaster method to determine the concentration of differentiable parasite forms per gram of faeces. Two grams of faeces were homogenised in $28 \mathrm{ml}$ of zinc sulphate flotation solution (specific gravity 1.200) and filtered through double cotton gauze. Concentration of parasite forms was estimated by screening two complete McMaster flotation chambers with a lower detection level of 15 parasite forms per gram. Separate concentrations of Nematodirus spp., Marshallagia spp., Strongyloides spp. and Trichuris spp. eggs per gram (epg) were determined, whereas the epg of the remaining gastrointestinal nematode genera were estimated as a whole (hereafter strongyle-EPG). Additionally, the concentration of protostrongylid lungworm larvae (lpg) and the presence/absence of Moniezia spp. eggs were also recorded.

Ewes of each mating batch were randomly distributed into two experimental groups: the non-treated control group and the treated group, in which ewes were dewormed by simultaneous administration of ivermectin (Tizoval $^{\circledR}, 0.2 \mathrm{mg} \mathrm{kg}$ live weight) and netobimine (Hapasil $^{\circledR}, 20 \mathrm{mg} \mathrm{kg}$ live weight) by subcutaneous and oral route, respectively. Deworming was conducted on the day following coprological examinations, i.e., in the third day after sampling. The two experimental groups remained together in the same batch (without any physical barrier) in order to recreate the management performed in a commercial farm when a TST is implemented.

To verify the efficacy of deworming, faecal samples were again obtained from all the ewes 14 days after treatment and individually examined with the same McMaster method. In addition, bulk faecal cultures for each experimental group were kept in an incubator at $23^{\circ} \mathrm{C}$ for 13 days. On day 14, the infective larvae were harvested and identified $(\approx 100$ larvae per coproculture) using identification keys for ruminant nematodes (MAFF, 1986).

Body condition score of all ewes was recorded five weeks (BCSm1) and one day (BCSm2) before the rams were introduced into the mating batch. To determine the BCS, the same person scored all the animals during the survey using a scale from 1 to 5 (Russel et al., 1969) $(1=$ emaciated; $5=$ fat) at 0.25 precision steps.

The pre-partum sampling was performed five weeks before the estimated date for first planned parturitions. The procedure was identical to the one followed in the pre-mating period. After parasitological 
analyses, ewes were also randomly distributed into two groups: the non-treated control group and the treated group, whereas BCS was recorded five weeks (BCSp1) and three days (BCSp2) before the estimated date for first parturitions.

During lambing, date of parturition and number of new-born lambs were individually recorded for all ewes. At the moment of birth, newborns were sexed and weighed, indicated as being born alive or dead, and individually identified by numbered ear tags. If death of lambs occurred during lactation or if a lamb was separated from its dam and raised with artificial lactation, the date of the event was recorded. Finally, the date and weight of lamb at weaning was registered.

\subsection{Statistical analyses}

The goal of the analytical approach was to determine the relationships among coprological parameters, treatment indicators, deworming and reproductive sheep efficiency at the level of the individual. Mating batches, however, could be a putative confounding factor due to intrinsic variations between them regarding management, season or other uncontrolled factors. To avoid overlap of the putative confounding effect with the parameter relationships during selection of the predictor variables, batches were not included in the initial models; that is, variance/deviance potentially explained by batches was left to the model error term. Nevertheless, to test that the batch effect was not a crucially confounding factor in any analysis, after the final model was obtained, it was re-fitted, with the batch being added as a random categorical predictor variable to corroborate that the batch did not show a statistically significant relationship with the dependent variable and that the regression parameters of the remaining predictor variables were similar in magnitude and sign.

\subsubsection{Pre-mating period}

Changes in BCS were evaluated by adjusting a linear regression model in which BCSm2 was the dependent variable, whereas the age, genotypic group, the main effect, two-way interactions of treatment, BCSm1 and the coprological parameters were included as predictor variables in the initial model.

Putative factors associated with apparent fertility determined at the moment of parturition were explored by fitting a logistic regression model with log-link function. The dependent variable was coded as 0 (no lambing) and 1 (lambing). Age, genotypic group, the main effects, two-way interactions of treatment, BCSm1 and the coprological parameters were included as predictor variables in the initial model.

In a similar way, pre-mating factors related to litter size (estimated as the number of new-borns independently if they were born alive or dead), and variation of the time $\left(\mathrm{T}_{\mathrm{dm}}\right)$ elapsed from the date of introduction of rams to parturition (as surrogate of the "delay-in-mating") were explored by fitting linear and logistic (with log-link function) regression models, respectively. The same predictor variables as in the former analysis were included in the initial models.

To reduce the confounding effect of the type of insemination, only data of naturally mated ewes were used in the apparent fertility, litter size (log-transformed) and delay-in-mating analyses.

\subsubsection{Pre-partum period}

Changes in BCS were evaluated by adjusting the linear regression models in which the BCSp2 was the dependent variable. As predictors, the age, genotypic group, the time $\mathrm{T}_{\mathrm{dm}}$ (as surrogate of time to parturition), litter size and its interaction with the treatment group, the main effects, two-way interactions of treatment, BCSp1 and the coprological parameters, were included as predictor variables in the initial model.

\subsubsection{Parturition and lactation period}

Factors associated with lamb live weight at birth were explored by fitting a linear regression model in which age, genotypic group, BCSp1, litter size, sex of the lamb, the coprological parameters, the treatment and its two-way interactions with BCSp1 and coprological parameters were included as predictor variables in the initial model.

Similarly, putative factors associated with lamb mortality were explored by fitting logistic regression models with log-link function to mortality data from birth to $24 \mathrm{~h}$ after parturition, including lambs born dead (peri-partum mortality) and from $24 \mathrm{~h}$ after parturition to weaning (mortality during lactation). In both models, the predictor variables included in the initial model were the same as in the previous model fitted to lamb weight at birth. Data of lambs raised with artificial lactation were excluded from the mortality analyses. Mortality rates were compared using odds ratios.

Finally, factors related to lamb growth during lactation were explored by fitting a linear regression model to mean live weight gain $(\mathrm{g} /$ day) of lambs from birth to weaning (MLG). As predictors, the variables included in the initial model were the same as in previous models, with the addition of other variables: the time (in days) elapsed from birth to weaning $\left(\mathrm{T}_{\mathrm{w}}\right)$, as a way to control variations of the growth gradient of lambs associated with the time elapsed until weaning, and the number of lambs raised by the ewe during lactation, as a surrogate of the milk availability per lamb during this period. This variable was estimated as the number of lambs weaned by the ewe plus the value $T_{d} / T_{w}$ estimated for every lamb dead or raised with artificial lactation, with $\mathrm{T}_{\mathrm{d}}$ being the time (in days) elapsed from birth to date when the lamb in question died or was transferred to artificial lactation.

For all analyses, a backward stepwise selection procedure was applied. Variables were retained in the final model if the $\mathrm{F}$ (linear regression) or Wald (logistic regression) statistic $\mathrm{p}$ value was $\leq 0.1$. Coprological parameters were lnp1-transformed (natural logarithm plus 1) except for the Moniezia spp. that was included as a binary variable. For categorical factors, the Rasa Aragonesa breed and non-treated group (control) were used to establish baseline levels for the genotypic group and deworming treatment, respectively.

To graphically explore the relationships detected by regression models between coprological parameters, BCS, treatment group and productive parameters, the final models obtained were re-fitted, while excluding the treatment variable and/or its interactions with other variables and allowing separate estimation of partial residues from control and treated ewes. Cumulative means and their $95 \%$ confidence intervals $(95 \% \mathrm{CI})$ of partial residuals were calculated from low to high BCS values and used to graphically estimate BCS threshold values that maximised differences between both groups.

In the case of exploring threshold values for lnp1-transformed coprological parameters, since they followed a negative binomial distribution (NBD) (with most of the animals exhibiting low values and few animals exhibiting high values), a two-degrees of freedom cubic spline smoother function was separately fitted to partial residues estimated from the control and treated ewes (Schimek, 2000). This was done to avoid results mainly determined by aggregations of coprological variables in the experimental flock and, subsequently, to obtain a more general pictures relevant to a wider spectrum of flocks. In those cases, given that the lowest detection level of the coprological analyses was $15 \mathrm{epg}$ and that $\operatorname{lnp} 1(15)=2.77$, graphs were interpolated in a range from $>0$ to $<2.77$.

\subsubsection{Modelling distribution of the FECs}

After threshold FEC values had been estimated as criteria to target anthelmintic treatment, a modelling exercise was performed to evaluate the relationships between flock mean FEC and the proportion of ewes within the flock with a FEC higher than the estimated threshold. Individual FECs in a flock were modelled using an NBD with two parameters: the arithmetic mean FEC of the flock $(m)$ and the inverse degree of aggregation ( $k$ ) (Dobson et al., 2009). In a first step, $k$ parameter was estimated for each experimental batch from individual strongyle-EPG values, with the minimum and maximum $k$ values estimated being 0.22 and 1.32, respectively. These two values were 
considered as representative of the range in variation for the degree of aggregation of FECs within flocks in the study area. Second, Monte Carlo simulations were conducted to successively model FEC distributions for all the combinations of the two $k$ values and theoretical mean flock FEC values of $m$ ranging from 50 to 1500 epg. Each combination was iterated for 1000 estimations, and the mean percentage of ewes with an FEC higher than the threshold value for each $m$ value was calculated. The random variables (NBDs), $k$ estimation and Monte Carlo simulations were generated using PopTools (CSIRO, Australia) within Excel (Microsoft, Inc., USA).

\section{Results}

Samples were characterised by a high variability covering a wide range of values for BCS and coprological parameters. Mean values and their standard deviations estimated for every mating batch at the premating and pre-partum periods and for non-treated and treated ewes are shown in Supplementary Table S1 and S2 respectively.

Body condition score ranged from 1.0 to 4.5, indicating the existence both of emaciated and fat individuals. Overall mean values estimated for non-treated and treated ewes showed that the mean BCS increased during the pre-mating period, both in the non-treated and the treated group, indicating an improvement of the physiological condition of ewes as a consequence of supplementation with the feed mixture. In contrast, the mean BCS decreased during the pre-partum period (Supplementary Table S2)

Strongyle-EPG and protostrongylid-LPG were the groups more frequently identified, with $83.2 \%$ and $27.2 \%$ prevalence in the premating sampling and $86.2 \%$ and $22.4 \%$ in the pre-partum sampling, respectively. The maximum range value for strongyle-EPG was 23,880 and 22,425 epg in the pre-mating and pre-partum sampling respectively, whereas for protostrongylid-LPG, it was 2130 and $810 \mathrm{lpg}$. Nevertheless, their mean values per batch showed important variations (Supplementary Table S1). In contrast, the presence of Nematodirus spp. (overall range 0-240 epg), Marshallagia spp. (overall range 0-15 epg), Strongyloides spp. (overall range 0-600 epg), Trichuris spp. (overall range 0-75 epg) and Moniezia spp. was marginal, with prevalences lower than $5 \%$ in all cases. For simplification, only the mean values of strongyle-EPG and protostrongylid-LPG are presented in Supplementary Table S1 and S2.

The genera identified by faecal cultures included Teladorsagia spp., Haemonchus spp., Chabertia spp. and Trichostrongylus spp. Teladorsagia spp. was the dominant genus, present in all batches in the pre-mating and pre-partum periods, with a relative abundance ranging from 7 to $100 \%$. Haemonchus spp. was identified in 8 and 7 batches in the premating and pre-partum periods, respectively, with relative abundances ranging from 3 to $90 \%$, whereas Chabertia spp. was identified in 7 and 4 batches, respectively, with relative abundances ranging from 2 to 14 $\%$. Finally, Trichostrongylus spp. was only identified in 4 and 5 batches, respectively, with relative abundances ranging from 2 to $10 \%$.

Overall, for treated ewes, the efficacy of the anthelmintic treatment was adequate, with faecal count reductions of $99 \%$ or higher in strongyle-EPG and protostrongylid-LPG in both the pre-mating and prepartum periods. Anthelmintic efficacy was also high for the remaining genera identified in the coprological analyses, whose prevalences were $0 \%$ after deworming in all cases.

\subsection{Pre-mating period}

Final model fitted to explore changes in BCS is shown in Table 1. Genotypic group and age were factors related to changes in BCS. Regarding coprological variables, only lnp1(strongyle-EPG) was retained in the model, showing a direct relationship with BCS increases. Meanwhile, interaction of treatment with $\operatorname{lnp} 1$ (strongyle-EPG) was also retained in the model. That is, treatment was positively associated with increases in BCS but, for dewormed ewes, the gains in BCS were greater
Table 1

Regression coefficients \pm SE and p values of predictor variables retained in the final regression model fitted to explore changes in body condition score (BCS) in pre-mating period.

\begin{tabular}{llll}
\hline Linear model fitted to BCSm2 $\left(\mathrm{R}^{2}=0.37 ;\right.$ d.f. $\left.=976 ; \mathrm{F}=95.03 ; \mathrm{p}<0.001\right)$ \\
\hline & $\begin{array}{l}\text { Regression } \\
\text { coefficients } \pm \mathrm{SE}\end{array}$ & $t$ & $\mathrm{p}$ value \\
& & & \\
\hline Genotipic group & $-0.14 \pm 0.04$ & -3.63 & $<0.001$ \\
ROA $^{\circledast}$ & $0.08 \pm 0.03$ & 2.28 & 0.022 \\
F1 & $-0.02 \pm 0.01$ & -2.63 & 0.009 \\
Age & $0.55 \pm 0.02$ & 22.12 & $<0.001$ \\
BCSm1 & $0.03 \pm 0.01$ & 4.09 & $<0.001$ \\
Lnp1(strongyle-EPG) & $0.01 \pm 0.003$ & 1.79 & 0.073 \\
Treatment x Lnp1(strongyle- & & & \\
$\quad$ EPG) & & &
\end{tabular}

BCSm1: BCS recorded just before the treatment, five weeks before the rams were introduced into the mating batch; BCSm2: BCS recorded one day before the rams were introduced.

when strongyle-EPG was higher.

No coprological parameter was found to be statistically associated with apparent fertility. The final logistic model only retained the genotypic group, treatment and treatment $\mathrm{x}$ BCSm1 interaction, showing that the treatment increased the apparent fertility, but the magnitude of this effect was inversely associated with BCSm1 (Supplementary Table S3).

Cumulative means of partial residuals from low to high BCSm1 values are shown in Fig. 1 to illustrate this relationship. The greatest difference in apparent fertility between the control and dewormed ewes occurred at threshold BCSm $1<2.75$ because the difference consistently decreased when ewes with higher BCSm1 were cumulated.

When the logistic regression analysis was repeated, after the BCSm1 variable had been categorised into two levels $-\mathrm{BCSm} 1<2.75$ and BCSm1 $\geq 2.75$ (baseline level)- the final model (Supplementary Table S3) also retained the genotypic group, the categorised BCSm1 and the treatment $\mathrm{x}$ categorised $\mathrm{BCSm} 1$ interaction, indicating the usefulness of BCSm1 $<2.75$ as the potential threshold value. At an observational level, the apparent fertility ( \pm SE) within the control group was 0.76 (0.02) and 0.87 (0.02) for ewes with BCSm1 $<2.75$ and BCSm $1 \geq 2.75$, respectively, whereas it was $0.84(0.03)$ and $0.84(0.03)$ within the dewormed group, respectively; that is, the apparent fertility was $8 \%$ higher in dewormed-BCSm $1<2.75$ ewes than in the control ones.

The final regression model adjusted to log-transformed litter size

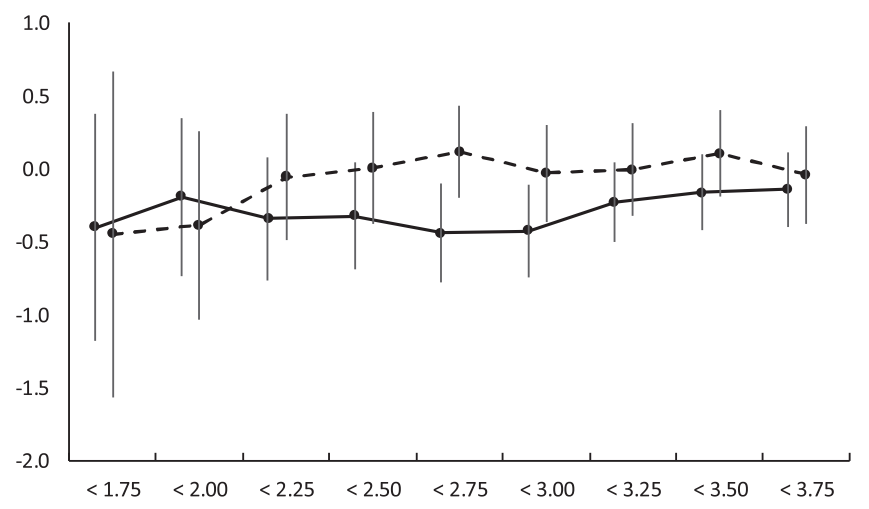

Fig. 1. Mean (95\% CI) of partial residuals of logistic model fitted to apparent fertility after controlling the effects of genotypic group. Mean is cumulated from low to high BCS (horizontal axis) estimated at pre-mating sampling, five weeks before the rams were introduced into the mating batches (BCSm1). Major difference arose between the control and treated groups when ewes with BCSm1 $<2.75$ were cumulated. Continuous line, non-treated control group; Dashed line, treated group. 
(Supplementary Table S3) only retained the genotypic group and the BCSm1 as predictor variables. That is, litter size mainly depended on the genotypic group, which was the highest for the F1 ewes and was directly related to BCSm1. No statistically significant association with treatment or any coprological parameter was found.

The time elapsed from the date of introduction of rams to parturition $\left(\mathrm{T}_{\mathrm{dm}}\right)$ ranged from 139 to 182 days, showing a bimodal distribution corresponding to ewes becoming pregnant at the first and second ovulation cycle. Classifying ewes with $\mathrm{T}_{\mathrm{dm}} \leq 160$ or $\mathrm{T}_{\mathrm{dm}}>160$ days as getting pregnant at the first or second ovulation cycle, respectively, the logistic final model (Supplementary Table S3), when fitted with baseline level $\mathrm{T}_{\mathrm{dm}}>160$, retained the genotypic group, the age, the treatment and the treatment $\mathrm{x}$ BCSm1 interaction. Therefore, in addition to showing differences among the genotypic groups, the model showed that, among lambing ewes, becoming pregnant on the first ovulation cycle was inversely related to age but positively associated with deworming, although, again, this positive relationship was lower for ewes with higher BCSm1 values. Raw observational data were in agreement with modelling results because, for individuals with BCSm1 $<2.75$, the proportion (SE) of lambing ewes becoming pregnant during the first ovulation cycle was $0.78(0.03)$ and $0.85(0.03)$ for the control group and dewormed group, respectively, whereas it was similar among ewes with $\mathrm{BCSm} 1 \geq 2.75$, at $0.81(0.03)$ and $0.82(0.03)$ within the control and dewormed group respectively.

\subsection{Pre-partum period}

Final model fitted to explore changes in BCS is in Table 2. Age and litter size were associated with changes in BCS since younger ewes and ewes gestating more foetuses had a higher decrease in their BCS during pre-partum. The relationships found for $\mathrm{T}_{\mathrm{dm}}$ with BCS indicated that ewes getting pregnant earlier exhibited a higher decrease in BCS, in agreement with a more advanced gestation. Lnp1(strongyle-EPG) was also retained in the final model, showing a negative relationship with BCS changes; that is, the decrease in BCS was greater with the higher $\operatorname{lnp} 1$ (strongyle-EPG), with no difference between the control and treated ewes.

\subsection{Parturition and lactation period}

The regression model fitted to the lamb live weight at birth (Supplementary Table S4) showed that this variable was associated with the genotypic group. Lamb live weight at birth was also directly related to age and inversely to BCSp1 and litter size. Lamb sex also determined the weight since females were lighter than males.

Regarding coprological parameters, only lnp1(strongyle-EPG) was retained, showing an inverse relationship with the weight of lambs at birth. Finally, the interaction of treatment $x \operatorname{lnp} 1$ (strongyle-EPG) was

\section{Table 2}

Regression coefficients $\pm \mathrm{SE}$ and p values of predictor variables retained in the final regression model fitted to explore changes in body condition score (BCS) in pre-partum period.

\begin{tabular}{lllc}
\hline Linear model fitted to BCSp2 $\left(\mathrm{R}^{2}=0.43 ;\right.$ d.f. $\left.=511 ; \mathrm{F}=77.55 ; \mathrm{p}<0.001\right)$ \\
\hline & Regression coefficients $\pm \mathrm{SE}$ & $t$ & $\mathrm{p}$ value \\
\hline Age & $-0.09 \pm 0.01$ & -8.63 & $<0.001$ \\
Litter size & $-0.09 \pm 0.03$ & -2.82 & 0.005 \\
$\mathrm{~T}_{\mathrm{dm}}$ & $0.01 \pm 0.002$ & 3.09 & 0.002 \\
$\mathrm{BCSp} 1$ & $0.56 \pm 0.03$ & 16.01 & $<0.001$ \\
Lnp1(strongyle-EPG) & $-0.02 \pm 0.01$ & -1.89 & 0.059 \\
\hline
\end{tabular}

BCSp1: body condition score recorded just before the treatment, five weeks before the estimated date for first parturitions; BCSp2: body condition score recorded three days before the estimated date for first parturitions; $\mathrm{T}_{\mathrm{dm}}$ : time elapsed from the date of introduction of rams to parturition as surrogate of delay-in-mating.
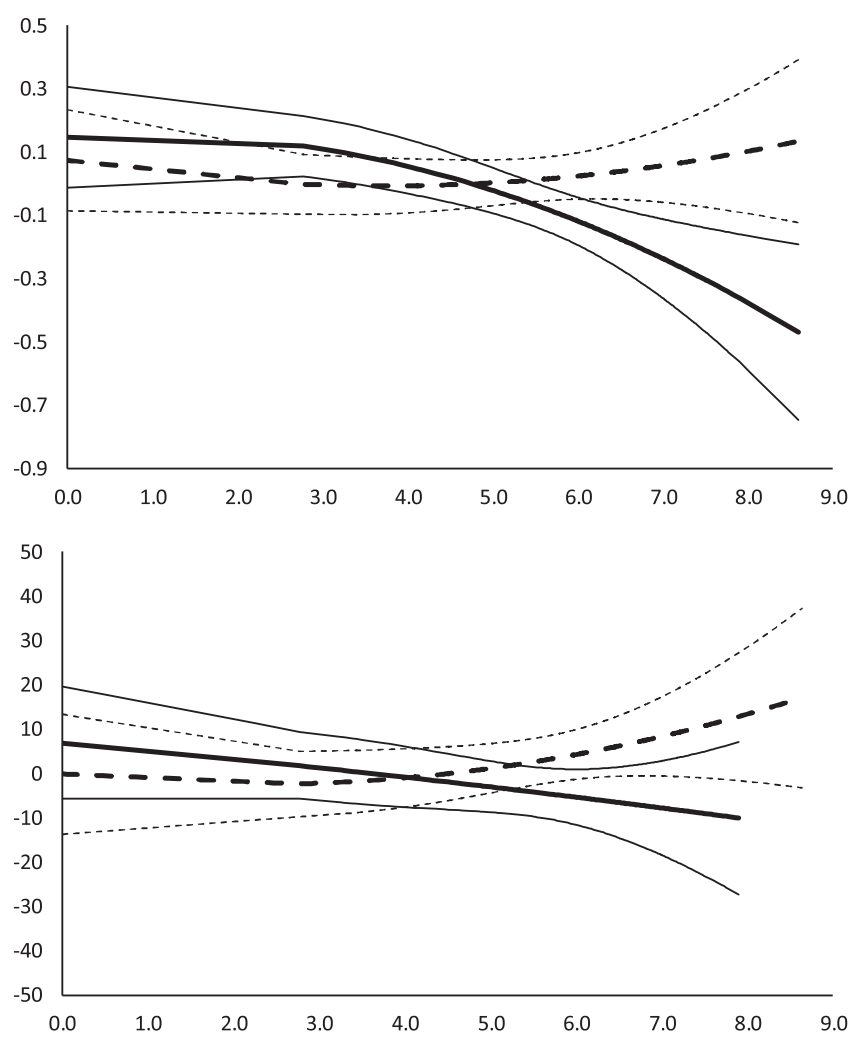

Fig. 2. Cubic spline smoother function (and $95 \% \mathrm{CI}$ ) fitted to partial residuals of regression models adjusted to the weight of the lambs at birth (upper graph) and mean live weight gain (MLG) (lower graph) during lactation as a function of lnp1(strongyle-EPG) (horizontal axes). Continuous line, non-treated control group; Dashed line, treated group.

also retained, suggesting that deworming had a positive effect on lamb weight, but this effect was higher with greater strongyle FEC value before deworming.

Graphical representation of the relationship among lamb weight, treatment and strongyle-EPG (Fig. 2, upper graph) showed that, whereas the treatment had an upper positive effect on the weight of lambs born from treated ewes with higher strongyle-EPG, for nontreated ewes, the weight of their lambs was negatively associated with this variable even at low egg elimination rates. Graphs for control and treated ewes overlapped at low egg elimination rates; however, from $\operatorname{lnp} 1$ (strongyle-EPG) $\approx 6$, which is equivalent to elimination rates $\approx$ 400 epg, both graphs clearly diverged, with no overlap of their $95 \%$ confidence intervals.

When the regression analysis was repeated, after the strongyle-EPG variable had been categorised into two levels - strongyle-EPG $>400$ epg and strongyle-EPG $\leq 400$ epg (baseline level) - the final model (Supplementary Table S4) also retained the categorised strongyle-EPG and the treatment $x$ categorised strongyle-EPG interaction, confirming the threshold value of 400 epg. Moreover, least square mean (SE) of weight of lambs at birth estimated from the model for the control group was $3.28 \mathrm{~kg}(0.04)$ and $3.06 \mathrm{~kg}(0.08)$ for lambs born from ewes with strongyle-EPG $\leq 400 \mathrm{epg}$ and strongyle-EPG $>400 \mathrm{epg}$ respectively, whereas it was $3.28 \mathrm{~kg}(0.04)$ and $3.25 \mathrm{~kg}(0.08)$ within the dewormed group, respectively.

Lamb peri-partum mortality rate ( \pm SE) was higher in lambs born from control ewes $(0.07 \pm 0.01)$ than in lambs born from dewormed ones $(0.05 \pm 0.01)$. The final logistic model obtained (Supplementary Table S4) only retained the litter size and the treatment, showing that mortality was higher among lambs belonging to bigger litters and that the odds ratio for dying was 1.24 times (90\% CI: 1.001-1.53) greater for lambs born from control ewes in comparison to those born from 


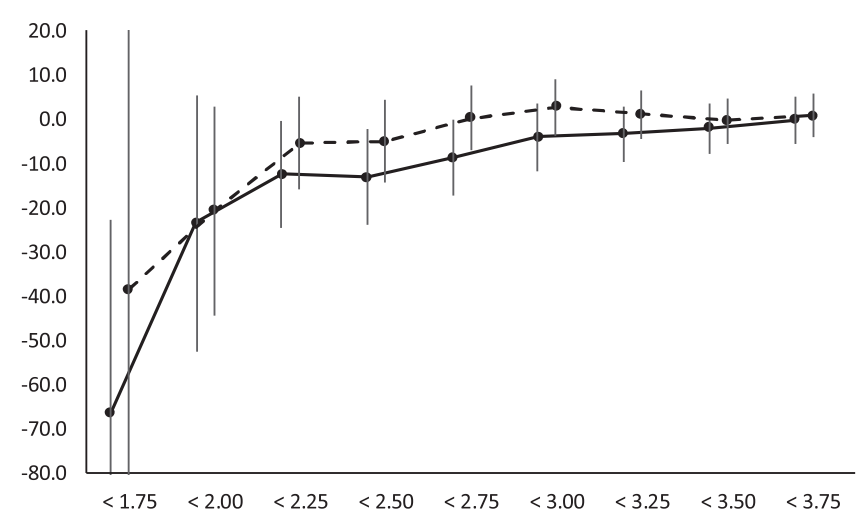

Fig. 3. Mean ( $95 \% \mathrm{CI})$ of partial residuals of regression model fitted to mean live weight gain ( $\mathrm{g}$ /day) of lambs from birth to weaning (MLG). Mean is cumulated from low to high BCS (horizontal axis) estimated at pre-partum sampling, five weeks before the estimated date for first parturitions (BCSp1). Continuous line, non-treated control group; Dashed line, treated group.

dewormed ewes.

Regarding mortality from $24 \mathrm{~h}$ after birth to weaning, the logistic final model (Supplementary Table S4) only retained the genotypic group and the litter size, showing that mortality during lactation was lower for lambs born from F1 ewes but higher for those belonging to litters with more lambs. However, neither deworming nor coprological parameters were found to be related to mortality at this stage.

Finally, following the final model adjusted to MLG (Supplementary Table S5), this parameter was dependent on the genotypic group and the lamb sex, since females had lower MLG than males. On the other hand, MLG was directly related to BCSp1 (Fig. 3) but was inversely associated with ewe age, litter size, the number of lambs raised and the time $\mathrm{T}_{\mathrm{w}}$ elapsed from birth to weaning. Finally, the interaction for treatment $x \operatorname{lnp} 1$ (strongyle-EPG) was also retained, indicating that deworming had a positive effect on lamb growth during lactation, but the magnitude of this effect was directly related to the strongyle FEC value of the ewe at deworming. The graphical examination of the relationship among the MLG, treatment and $\operatorname{lnp} 1$ (strongyle-EPG) (Fig. 2, lower graph) showed a pattern similar to that estimated for lamb weight at birth, also suggesting a divergence point for both groups (control and treated ewes) of approximate $\ln 1$ (strongyle-EPG) values 6-6.4 $(\approx$ 400-600 epg), although in this case, the nonexistence of weaned lambs born from non-treated ewes with the highest strongyle-EPG values probably entailed the widening and overlapping of confidence intervals.

As in the case of weight of lambs at birth, the regression analysis was repeated, after the strongyle-EPG variable had been categorised into two levels - strongyle-EPG $>400 \mathrm{epg}$ and strongyle-EPG $\leq 400$ epg (baseline level) - the final model (Supplementary Table S5) also retained the treatment $x$ categorised strongyle-EPG confirming that the benefit of deworming was higher in the growth of lambs born from ewes with strongyle-EPG $>400$ epg. Least square mean (SE) of MLG estimated from the model for the control group was $215.4 \mathrm{~g}$ /day (3.0) and $204.8 \mathrm{~g} /$ day (4.6) for lambs born from ewes with strongyleEPGp1 $\leq 400$ epg and strongyle-EPGp1 $>400$ epg respectively, whereas it was $213.7 \mathrm{~g} /$ day (3.3) and $224.3 \mathrm{~g} /$ day (4.3) within the dewormed group, respectively.

\subsection{Modelling FECs distribution}

Given that the range of 400-600 epg was identified to show the potential individual FEC threshold values for targeting treatment during the pre-partum period, a modelling exercise was carried out for both limits of the range (Fig. 4). The figure shows that the percentage of ewes with FEC higher than 400 or 600 epg barely reaches $10 \%$ for flock mean $\mathrm{FEC}$ values $\approx 200 \mathrm{epg}$, whereas that percentage at flock mean

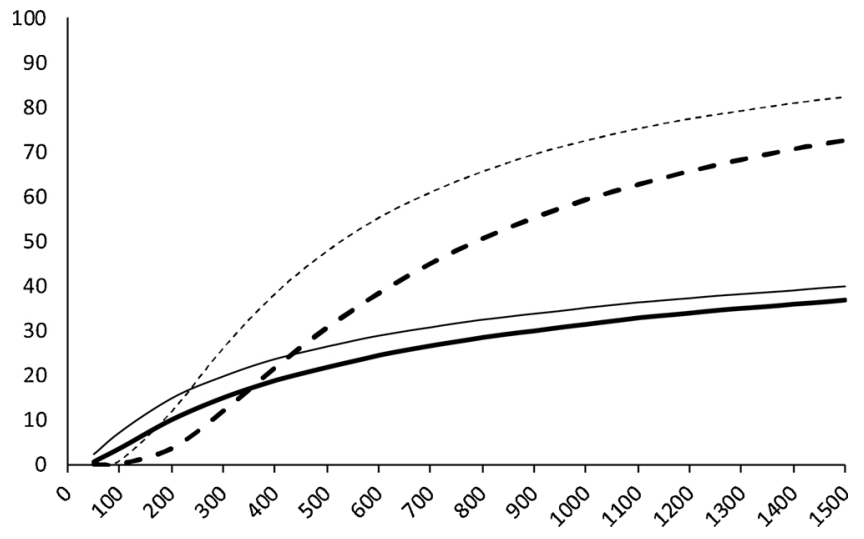

Fig. 4. Percentage (vertical axis) of ewes with FEC $>400$ epg (thin lines) or FEC $>600$ epg (thick lines) as a function of mean FEC of the flock (horizontal axis). Simulations have been done for two values of the inverse degree of aggregation $(k)$ indicative of FECs aggregation within the flock: High aggregation $(k=0.22)$, continuous lines; Low aggregation $(k=1.32)$, dashed lines.

FEC $\geq 400$ epg shows an increase in value by $\approx 20 \%$ or higher in all cases, especially in scenarios of low aggregation, where the percentage of ewes with FEC $>400$ epg reaches values of $50 \%$. At mean flock FEC higher than $400 \mathrm{epg}$, the percentage of ewes whose productivity would be potentially affected by GIN infection increases considerably and, therefore, this would imply remarkable productive loses for the flock.

\section{Discussion}

The difficulty in quantifying relationships between parasitic infections and productive performance of sheep under field conditions increases in epidemiologic scenarios in which parasitic infections are subclinical, since the pathophysiological effects of infection can be largely balanced by host nutrition and its complex interactions with nutrient metabolism and immunological response of the host (Adams and Liu, 2003; Knox et al., 2006). The difficulty increases when concurrent multispecies GIN infections occur, since the pathophysiological effects of the several species can overlap in different ways. Moreover, using FEC as an indicator of the level of parasitism or pathogenic effect on the host adds variability, since egg elimination rates do not only depend on parasite numbers (Sargison, 2013). Caution, therefore, is necessary, when considering that the quantification of the impact of the parasites determined by statistical models is realistic and directly extrapolated to commercial farms.

In the present study, only strongyle-EPG, comprising combined FECs of Teladorsagia spp., Haemonchus spp., Chabertia spp. and Trichostrongylus spp. was found to be related to negative effects on physiology and productivity of ewes, confirming that this group, characterised by high prevalence and infection intensities in the study area (Calvete et al., 2012) must be one of the main targets of the parasite control programmes in this type of productive and parasitological scenario. The fact that no statistical relationship was found for the remaining gastro-intestinal parasite groups probably was not due to a lack of pathogenicity but to a low prevalence and infection intensity in the experimental flock. Whereas, in the case of lungworms, although their prevalence was moderate, no negative relationship was found, perhaps because their pathogenicity is lower or because their effect was masked by noise factors not controlled in the analyses.

On the other hand, it is known that ewes should have a BCS in the range of 2.5-3.5 at the start of the mating season and should maintain it in early pregnancy to display optimal reproductive performances (Kenyon et al., 2014). For this reason, the improvement of a nutritional regime is usually initiated at least 35 days before the start of the mating. However, since the reduction in the efficiency of absorbed nutrients subsequent to parasitism by gastrointestinal helminths is the 
most significant energy-drain of clinically healthy ewes, the period immediately before and around conception is a potentially vulnerable time for detrimental effects of parasites (Fthenakis et al., 2015). Accordingly, deworming before mating has already been demonstrated to improve fertility or apparent fertility (Lewis, 1975; Mackay, 1980; Pandey et al., 1984) and litter size (Murray et al., 1971; Lewis, 1975; Chaudhry et al., 1987; Mavrogianni et al., 2011), which presumably are related to higher cycling rate, amelioration of genital system functionality and an increased number of ovulations.

The present results clearly agree with former studies. Results have shown that anthelmintic treatment implies taking better advantage of benefits in the improved nutritional regime, since dewormed ewes experimented a quicker amelioration of their physiological condition from treatment to mating, as supported by a higher gain in BCS than in the control ewes. Moreover, the gain of BCS among dewormed ewes was directly related to strongyle-EPG, suggesting that, if it is assumed that greater parasite intensity occurs with higher EPG, the benefit of deworming was more marked for the more parasitized ewes.

As a consequence, deworming improved reproductive efficiency, since anthelmintic treatment increased apparent fertility of ewes with BCS $<2.75$, as well as the proportion of them that got pregnant at first ovulation, probably because deworming improved conception rates as a secondary response to the quick amelioration of body condition. Previous studies, however, have found that anthelmintic drenching before mating reduces foetal losses (Mackay, 1980; Pandey et al., 1984); therefore, the apparently augmented fertility could also be subsequent to a higher foetal losses rate (or suboptimal ova survival) in control ewes that conceived during the first ovulation cycle. Given that no early pregnancy diagnostic or miscarriage recording was performed in the present study, elucidation of the factor was related to apparent fertility improvement is not possible.

Interestingly, no association of apparent fertility with any coprological parameter was found, though strongyle-EPG and its interaction with treatment was related to BCS variation. This lack of relationships, however, may be secondary to both parasitological scenario, dominated by subclinical infections and the interactions between nutrition improvement and reproductive physiology of ewes before mating, which may have masked any relationship between FEC and apparent fertility.

During the pre-partum period, the last stage of pregnancy is a metabolically demanding time due to foetal requirements and the need to prepare colostrum and milk production (Fthenakis et al., 2012). As a consequence, deworming in the pre-partum period has been also associated with higher live weight of lambs at birth (Mackay, 1980; Nilsson and Johnelius, 1986; Fthenakis et al., 2005), lower lamb mortality (Mackay, 1980; Pandey et al., 1984; Chaudhry et al., 1987) and improved lamb growth (Sweeny et al., 2012).

The results of the present study show that FEC, in this case the strongyle-EPG, was associated with a detrimental effect on the evolution of BCS at the last stage of pregnancy but no effect of treatment was detected. Nevertheless, anthelmintic treatment improved productive parameters, such as the weight of lambs at birth, lamb survival and lamb growth during lactation. Lamb mortality was only reduced during peri-partum, probably associated with higher weight at birth of lambs born from drenched ewes. The improvement of both lamb weight at birth and MLG was directly related to strongyle-EPG values, showing that lambs most benefited by treatment were those born from ewes excreting $\approx 400$ epg or higher, confirming the usefulness of this threshold FEC value for selective treatment at this stage. Meanwhile, BCS at pre-partum sampling was found to be inversely related to the weight of lambs at birth, probably as a consequence of the trade-off between ewe body reserves and growth of foetuses during the last stage of pregnancy, and it was positively related to MLG, probably because it is associated with a higher milk yield of ewes in better body condition. However, no statistical interaction between treatment and BCS was found, making it difficult to identify any potential BCS threshold for selective treatment at this period.
The present results imply that the implementation of TST during the pre-mating and pre-partum periods can increase the productivity of sheep flocks in areas where subclinical GIN infections are dominant. These results clearly indicate that, during the pre-mating period, BCS should be the criterion used for target treatment at the individual level, with ewes showing BCS $<2.75$ being dewormed. This BCS value, therefore, could be stated as threshold criterion to optimize the tradeoff between productivity and TST implementation in pre-mating. Establishing a decision-making criterion for TST during the pre-partum period, however, is not so evident since strongyle-EPG was the main variable associated with effects of treatment on productivity parameters. Deworming ewes with strongyle-EPG above 400 epg has been shown to be an adequate criterion to optimize productivity, but individual FECs are not a practical option to be implemented in the field. Consequently, only mean FECs at the flock level could be derived as a decision-making criterion to prescribe TT (instead of TST) to all animals during the pre-partum period.

Thereby, under the light of the FEC modelling, for flock mean FEC values lower than $\approx 200 \mathrm{epg}$, anthelmintic treatment of all ewes could be unnecessary, since the percentage of ewes whose productive efficiency would be potentially affected by GIN infection is very low. However, at higher values, and especially at $400 \mathrm{epg}$, this percentage increases and could imply appreciable productive loses if deworming is not performed. Raw observational data supported these results because, in batches with mean FEC > 400 epg (batches 2 and 4; Supplementary Table S1), the mean weight of lambs at birth (SD) was $3.31 \mathrm{~kg}(0.76)$ and $3.65 \mathrm{~kg}(0.85)$ for the control and dewormed group respectively, whereas it was similar in batches with mean FEC $<200$ epg (batches 1,7 and 10), at $3.28 \mathrm{~kg}(0.87)$ and $3.28 \mathrm{~kg}(0.84)$ within the control and dewormed group respectively. Something similar occurred with MLG. In batches with mean FEC > 400 epg, the mean MLG (SD) was $212.8 \mathrm{~g} /$ day (59.1) and $220.1 \mathrm{~g} /$ day (50.9) for the control group and dewormed group, respectively, whereas it was similar in batches with mean FEC < $200 \mathrm{epg}$, at $210.4 \mathrm{~g} /$ day (67.2) and $209.0 \mathrm{~g} /$ day (52.0) within the control and dewormed group respectively. This threshold mean FEC value $(\approx 400 \mathrm{epg}$ ) is highly similar to arbitrary thresholds empirically derived from field observations (Gallidis et al., 2009; Rinaldi and Cringoli, 2012) or with categorisation of diagnostic results published in the technical manual for sustainable worm control strategies for sheep (SCOPS) (Abbot et al., 2012), supporting the results of the present approach.

In this way, the use of flock mean FEC would be useful as a decisionmaking criterion to prescribe TT during the pre-partum period in flocks in the study area, but TTs, however, do not cover the main goal of the TSTs: to maintain parasites in refugia within untreated ewes and preserve anthelmintic efficacy. To solve this, and based on the results represented in Fig. 3, BCS as a decision-making criterion to target anthelmintic treatment in pre-partum period is appealing. In this way, although MLG was improved by treatment in relation to strongyle-EPG, as shown in Fig. 2 (lower graph), the differences between the control and the treated ewes were clearly minimised from BCS $\approx 3$ or higher (Fig. 3). Therefore, it could be argued that drenching ewes with BCS $<3$ could also be stated as a threshold value to target TSTs in the pre-partum period. This inference agrees with the results of Cornelius et al. (2014), who observed that ewes with BCS $<3$ at the pre-lambing periods showed greater probabilities for falling below BCS $=2$ after lambing, increasing the risk of production losses.

To illustrate the potential productive benefits of applying a TST using BCS as the sole decision-making criterion for deworming in both periods, five virtual flocks were generated using the data obtained from the experimental flock. To generate these flocks, data of individual ewes were grouped in function of the treatment strategy applied, and two of the major factors that affect profitability in sheep production, i.e. mean number and mean total weight of lambs weaned per ewe were calculated for each virtual flock (Table 3). For these estimations, BCS $<2.75$ was set as criterion to prescribe anthelmintic drenching, 
Table 3

Mean (SD) number of lambs weaned per ewe and mean (SD) total weight (kg) of weaned lambs per ewe estimated for every virtual flock. Virtual flocks were generated in function of the treatment strategy applied to individual ewes. The number of ewes per virtual flock (n) and the mean number of treatments per ewe are also included.

\begin{tabular}{lllll}
\hline Virtual flocks & $\mathrm{n}$ & Treatments/ewe & $\begin{array}{l}\text { Weaned } \\
\text { lambs/ewe }\end{array}$ & $\begin{array}{l}\text { Total weight of } \\
\text { weaned lambs/ewe }\end{array}$ \\
\hline Treated & 112 & 2 & $1.30(0.43)$ & $18.92(6.79)$ \\
TST & 167 & 0.62 & $1.29(0.48)$ & $18.98(6.18)$ \\
Pre-mating & 179 & 0.43 & $1.27(0.49)$ & $18.08(7.04)$ \\
Pre-partum & 198 & 0.32 & $1.23(0.47)$ & $17.12(6.16)$ \\
Untreated & 199 & 0 & $1.18(0.44)$ & $16.46(6.02)$ \\
\hline
\end{tabular}

Treated: all ewes were treated both in pre-mating and pre-partum period; TST: tactical selective treatment was applied in both periods. Ewes were treated if BCS < 2.75; Pre-mating: TST was only applied in pre-mating, all ewes remained untreated in pre-partum; Pre-partum: TST was only applied in prepartum, all ewes remained untreated in pre-mating; Untreated: all ewes remained untreated in both periods.

and the virtual flocks were representative of the following five scenarios: treated, in which all ewes were treated in both periods; untreated, in which all ewes remained untreated in both periods; TST, in which the tactical selective treatment was applied in both periods and, finally, pre-mating or pre-partum scenarios in which TST was only applied in pre-mating or pre-partum period respectively.

As expected, the highest and the lowest productive performances were reached by the treated and the untreated virtual flock respectively (Table 3), whereas intermediate results were estimated for pre-mating and pre-partum flocks. Implementation of TST in pre-mating period, however, seemed largely to improve productivity in comparison to prepartum TST alone, probably because of increased apparent fertility of ewes that were treated before mating. Finally, the TST virtual flock reached productive performances that were very similar to those estimated for the treated one, although the TST virtual flock additionally entailed a considerable reduction in mean number of treatments per ewe, which could result in an improvement in profitability. These estimates, therefore, suggested that the implementation of a TST using BCS as the sole decision-making criterion could result in an improvement in profitability and in an optimized productive efficiency on sheep farms of the area, in comparison to traditional deworming schemes based on seasonal blind strategic treatments.

Consequently, it seems that the generalised scheme of the TST derived from the present results to control negative impact of GIN infections should include the anthelmintic treatment of ewes with BCS lower than $\approx 3$ about five weeks before mating and lambing to both improve productivity and maintain parasite population in refugia. Additionally, if flock mean FEC were considered a secondary decision-making criterion, in the case of pre-lambing, drenching may be considered unnecessary if the flock mean FEC of strongyle eggs were lower than $\approx$ 200 epg.

Finally, although it is considered that this study is representative of scenarios with subclinical infections in which Haemonchus spp. make up only a moderate proportion of the GIN species and haemonchosis occurs in a chronic form, caution is recommended when applying threshold values, especially FEC ones, in commercial farms. Haemonchus spp. is the most pathogenic of the common nematodes of small ruminants but also presents by far the highest egg output rate. Given that in parasitological scenarios with concurrent multispecies GIN infections the relative abundance of species can fluctuate, threshold FEC values could vary and, probably, be higher when Haemonchus spp. predominates. In the present study, although the relative abundance of Haemonchus spp. varied widely, ranging from 0 to $90 \%$ among batches, additional statistical analyses (not shown in the article) failed to detect statistically significant associations between threshold FEC values and the relative abundance of this species. This lack of statistical significance, nevertheless, does not prove that the estimated threshold values are necessarily optimal for other similar parasitological scenarios, therefore, it is advised that FEC (and BCS) threshold values be calibrated to parasitological and farm-specific conditions to optimize its efficacy (Sargison, 2013).

\section{Conclusion}

The results show the potential utility of BCS as decision-making criteria to perform TSTs in flocks with subclinical concurrent multispecies GIN infections. The TSTs would bring important benefits, such as the improvement of productivity and a rational management of parasites in refugia, thereby preserving the future efficacy of anthelmintics. These advantages should be pivotal to overcome the reluctance of farmers to change the current GIN control schemes; however, given that every farm is different, the general TST scheme should be adapted, and the decision criteria calibrated to parasitological scenario and farmspecific conditions to optimize its efficacy.

On the other hand, TST can incur additional costs for veterinary services in such a way that TST profitability will not only depend on the infection itself but also on the farm-specific factors involving the production system, the input and output prices, and the scale of production (Charlier et al., 2014b). Therefore, further field studies should be conducted in order to evaluate both practicality, impact on parasitic infection and economic profitability of this TST scheme in commercial farms as a first step towards promoting its general adoption.

\section{Acknowledgements}

Funding for this project was provided by the RTA2013-00064-C0201 grant from the National Institute for Agricultural and Food Research and Technology (INIA). Special thanks go to J.A. Tanco and A. Guillén for their assistance in field and laboratory work and to Carmen Margolles for her support. Thanks go also to the two anonymous reviewers for their valuable comments.

\section{Appendix A. Supplementary data}

Supplementary material related to this article can be found, in the online version, at doi:https://doi.org/10.1016/j.vetpar.2019.109018.

\section{References}

Abbot, K.A., Taylor, M.A., Stubbings, L.A., 2012. Sustainable Worm Control Strategies for Sheep. A Technical Manual for Veterinary Surgeons and Advisors, SCOPS, fourth edition. Context Publishing. http://scops.org.uk/vets-manual.html.

Adams, N.R., Liu, S.M., 2003. Principles of nutrient partitioning for wool, growth and reproduction: implications for nematode parasitism. Aust. J. Exp. Agric. 43, 1399-1407.

Calvete, C., Calavia, R., Ferrer, L.M., Ramos, J.J., Lacasta, D., Uriarte, J., 2012. Management and environmental factors related to benzimidazole resistance in sheep nematodes in Northeast Spain. Vet. Parasitol. 184, 193-203.

Charlier, J., Morgan, E.R., Rinaldi, L., van Dijk, J., Demeler, J., Höglund, J., Hertzberg, H., Van Ranst, B., Hendrickx, G., Vercruysse, J., Kenyon, F., 2014a. Practices to optimise gastrointestinal nematode control on sheep, goat and cattle farms in Europe using targeted (selective) treatments. Vet. Rec. 175, 250-255.

Charlier, J., van der Voort, M., Kenyon, F., Skuce, P., Vercruysse, J., 2014b. Chasing helminths and their economic impact on farmed ruminants. Trends Parasitol. 30, 361-367.

Chaudhry, M.A., Anwar, A., Chaudhry, A., Malik, S.Z., 1987. Effect of anthelmintic administration on reproductive behaviour of naturally parasitized sheep. Pakistan Vet. J. 1, 44-45.

Chylinski, C., Cortet, J., Neveu, C., Cabaret, J., 2015. Exploring the limitations of pathophysiological indicators used for targeted selective treatment in sheep experimentally infected with Haemonchus contortus. Vet. Parasitol. 207, 85-93.

Cornelius, M.P., Jacobson, C., Besier, R.B., 2014. Body condition score a selection tool for targeted selective treatment-based nematode control strategies in Merino ewes. Vet. Parasitol. 206, 173-181.

Dobson, R.J., Sangster, N.C., Besier, R.B., Woodgate, R.G., 2009. Geometric means provide a biased efficacy result when conducting a faecal egg count reduction test (FECRT). Vet. Parasitol. 161, 162-167.

Fthenakis, G.C., Arsenos, G., Brozos, C., Fragkou, I.A., Giadinis, N.D., Gianenas, I., 
Mavrogianni, V.S., Papadopoulos, E., Valasi, I., 2012. Health management of ewes during pregnancy. Anim. Reprod. Sci. 130, 198-212.

Fthenakis, G.C., Mavrogianni, V.S., Gallidis, E., Papadopoulos, E., 2015. Interactions between parasitic infections and reproductive efficiency in sheep. Vet. Parasitol. 208, 56-66.

Fthenakis, G.C., Papadopoulos, E., Himonas, C., 2005. Effects of three anthelmintic regimes on milk yield ewes and growth of lambs. J. Vet. Med. A: Physiol. Pathol. Clin. Med. 52, 78-82.

Gallidis, E., Papadopoulos, E., Ptochos, S., Arsenos, G., 2009. The use of targeted selective treatments against gastrointestinal nematodes in milking sheep and goats in Greece based on parasitological and performance criteria. Vet. Parasitol. 164, 53-58.

Kenyon, F., Jackson, F., 2012. Targeted flock/herd and individual ruminant treatment approaches. Vet. Parasitol. 186, 10-17.

Kenyon, F., McBean, D., Greer, A.W., Burgess, C.G., Morrison, A.A., Bartley, D.J., Bartley, Y., Devin, L., Nath, M., Jackson, F., 2013. A comparative study of the effects of four treatment regimes on ivermectin efficacy, body weight and pasture contamination in lambs naturally infected with gastrointestinal nematodes in Scotland. Int. J. Parasitol. Drugs Drug Resist. 3, 77-84.

Kenyon, P.R., Maloney, S.K., Blache, D., 2014. Review of sheep body condition score in relation to production characteristics. N.Z. J. Agri. Res. 57, 38-64.

Knox, M.R., Torres-Acosta, J.F.J., Aguilar-Caballero, A.J., 2006. Exploiting the effect of dietary supplementation of small ruminants on resilience and resistance against gastrointestinal nematodes. Vet. Parasitol. 139, 385-393.

Lewis, K.H.C., 1975. Ewe fertility response to pre-mating anthelmintic drenching. N. Z. J. Exp. Agric. 3, 43-47.

Mackay, R.R., 1980. The effect of strategic anthelmintic treatment on the breeding performance of hill ewes. Vet. Parasitol. 7, 319-331.

MAFF, 1986. Manual of Veterinary Parasitological Laboratory Techniques. Ministry of Agriculture, Fisheries and Food. Technical Bulletin No 18, HMSO, London, U.K, pp. $2-67$.

Martínez-Royo, A., Jurado, J.J., Smulders, J.P., Martí, J.I., Alabart, J.L., Roche, A. Fantova, E., Bodin, L., Mulsant, P., Serrano, M., Folch, J., Calvo, J.H., 2008. A deletion in the bone morphogenetic protein 15 gene causes sterility and increased litter size in Rasa Aragonesa sheep. Anim. Genet. 39, 294-297.

Martínez-Valladares, M., Martínez-Perez, J.M., Robles-Perez, D., Cordero-Perez, C., Famularo, M.R., Fernandez-Pato, N., Castañon-Ordoñez, L., Rojo-Vázquez, F.A., 2013. The present status of anthelmintic resistance in gastrointestinal nematode infections of sheep in the northwest of Spain by in vivo and in vitro techniques. Vet. Parasitol. 191, 177-181.

Mavrot, F., Hertzberg, H., Torgerson, P., 2015. Effect of gastro-intestinal nematode infection on sheep performance: a systematic review and meta-analysis. Parasit.
Vectors 8, 557

Murray, J., Leaning, W.H.D., Martin, C.A., 1971. Pre-mating anthelmintic treatment of ewes and its effect on lambing performance. N. Z. Vet. J. 19, 1-4.

Nilsson, O., Johnelius, G., 1986. The effects of albendazol treatment on spring-rise in faecal nematode egg output and reproductive performance in the ewe. Nord. Vet. Med. 38, 74-79.

Pandey, V.S., Cabaret, J., Fikri, A., 1984. The effect of strategic anthelmintic treatment on the breeding performance and survival of ewes naturally infected with gastro-intestinal strongyles and protostrongylids. Ann. Rech. Vét. 15, 491-496.

Rinaldi, L., Cringoli, G., 2012. Parasitological and pathophysiological methods for selective application of anthelmintic treatments in goats. Small Rumin. Res. 103, $18-22$.

Rose, H., Rinaldi, L., Bosco, A., Mavrot, F., de Waal, T., Skuce, P., Charlier, J., Torgerson, P.R., Hertzberg, H., Hendrickx, G., Vercruysse, J., Morgan, E.R., 2015. Widespread anthelmintic resistance in European farmed ruminants: a systematic review. Vet. Rec. $176,546$.

Russel, A.J.F., Doney, J.M., Gunn, R.G., 1969. Subjective assessment of body fat in live sheep. J. Agric. Sci. 72, 451-454.

Sargison, N.D., 2013. Understanding the epidemiology of gastrointestinal parasitic infections in sheep: What does a faecal helminth egg count tell us? Small Rumin. Res. 110, 78-81.

Schimek, M.G. (Ed.), 2000. Smoothing and Regression: Approaches, Computations and Aplication. Wiley, New York 581 pp.

Sweeny, J.P.A., Robertson, I.D., Ryan, U.M., Jacobson, C., Woodgate, R.G., 2012. Impacts of naturally acquired protozoa and strongylid nematode infection on growth and faecal attributes in lambs. Vet. Parasitol. 184, 298-308.

Torres-Acosta, J.F.J., Pérez-Cruz, M., Canul-Ku, H.L., Soto-Barrientos, N., CámaraSarmiento, R., Aguilar-Caballero, A.J., Lozano-Argáes, I., Le-Bigot, C., Hoste, H., 2014. Building a combined targeted selective treatment scheme against gastrointestinal nematodes in tropical goats. Small Rumin. Res. 121, 27-35.

Valcárcel, F., Aguilar, A., Sánchez, M., 2015. Field evaluation of targeted selective treatments to control subclinical gastrointestinal infections on small ruminant farms. Vet. Parasitol. 211, 71-79.

Valvárcel, F., Meana, A., Sacristán, E., Uriarte, J., Calvete, C., Calavia, R., Martínez Valladares, M., Rojo-Vázquez, F.A., 2013. Small ruminants farm management practices in Spain and its infuence on the development of anthelmintic resistance. Rev. Ibero-Latinoam. Parasitol. 72, 151-163.

Van Wyk, J.A., 2001. Refugia - overlooked as perhaps the most important factor concerning the development of anthelmintic resistance. Onderstepoort J. Vet. Res. 68 $55-67$. 